\title{
Cellular manufacturing system design using a holonistic approach
}

\section{Selim Akturk \& Ayten Turkcan}

To cite this article: M. Selim Akturk \& Ayten Turkcan (2000) Cellular manufacturing system design using a holonistic approach, International Journal of Production Research, 38:10, 2327-2347, DOI: 10.1080/00207540050028124

To link to this article: http://dx.doi.org/10.1080/00207540050028124

$$
\text { 曲 Published online: } 14 \text { Nov } 2010 .
$$

Submit your article to this journal

$$
\text { Цll Article views: } 100
$$

Q View related articles $\leftarrow$

\footnotetext{
4 Citing articles: 40 View citing articles
} 
INT. J. PROD. RES., 2000, VOL. 38, NO. 10, 2327-2347

\title{
Cellular manufacturing system design using a holonistic approach
}

\author{
M. SELIM AKTURK广* and AYTEN TURKCANं
}

We propose an integrated algorithm that will solve the part-family and machinecell formation problem by simultaneously considering the within-cell layout problem. To the best of our knowledge, this is the first study that considers the efficiency of both individual cells and the overall system in monetary terms. Each cell should make at least a certain amount of profit to attain self-sufficiency, while we maximize the total profit of the system using a holonistic approach. The proposed algorithm provides two alternative solutions; one with independent cells and the other one with inter-cell movement. Our computational experiments indicate that the results are very encouraging for a set of randomly generated problems.

\section{Introduction}

Group technology (GT) is an innovative approach to batch-type production which seeks to rationalize small-lot production by capitalizing on the similarities that exist among component parts and/or processes. GT tries to bring the benefits of mass production to high variety, medium-to-low volume quantity production. GT has several benefits such as reduced material handling, work-in-process inventory, setup time and manufacturing lead time, and simplified planning, routing and scheduling activities. The application of GT to manufacturing is cellular manufacturing (CM) which is the physical division of the manufacturing facilities into production cells, representing the basis for advanced manufacturing systems such as just-in-time, flexible manufacturing system and computer integrated manufacturing. In the partfamily and machine-cell formation (PFMCF) problem, machines are grouped into cells to produce a group of parts having similar design attributes or manufacturing requirements.

Cellular manufacturing system (CMS) design problem is very important, but complex in nature. In literature, different approaches are proposed to solve the CMS design problem. Offodile et al. (1994) provide a comprehensive review of the CM literature. The most popular method is called the matrix formulation technique, which uses the binary machine-part incidence matrix as an input and process on it by rearranging rows and columns according to some measures until visible clusters are formed. The most common objective is to minimize the inter-cell movements and inter-cell material handling costs. Although, the real impact of inter-cell and intracell moves cannot be realistically determined without knowing the production volumes, operation sequences and cellular layout. For example, the parts with high volumes cause more moves, whereas an intermediate operation in a cell can cause

Revision received September 1999 Turkey.

$\dagger$ Department of Industrial Engineering, Bilkent University, 06533 Bilkent, Ankara,

* To whom correspondence should be addressed. 
two inter-cell moves while the first or the last operation causes only one move. Furthermore, the existing studies usually assume that the available machine capacities are enough for producing all the parts, consequently an infinite capacity may not be a realistic assumption in most real-life cases. Moreover, if an additional machine investment cost is justified then additional machines can be bought to reduce inter-cell material handling costs, and ultimately to achieve the cell independence. Therefore, the available machine capacities, processing times and production volumes should be included in the design process to calculate the required machine capacities. Several mathematical programming models are formulated for the PFMCF problem. These models usually cannot be solved in reasonable computation times, but they can be used to provide insights into the development of good heuristic methods. Local search heuristics such as simulated annealing, tabu search and genetic algorithms are proposed to solve the mathematical programming models (Heragu and Gupta 1994, Joines et al. 1996, and Vakharia and Chang 1997).

In several studies, it is assumed that each part has only one fixed route, except the studies by Choobineh (1988), Ho and Moodie (1996), Adil et al. (1996), Beaulieu et al. (1997) and Askin and Zhou (1998). When each part has only one routing, the creation of independent cells may not be possible without buying additional machines or allowing inter-cell moves. In most of the manufacturing firms, the parts usually have alternative routes. Furthermore, in the existing literature, the part-family formation process is usually made independent of the machine-cell formation process. In other words, there is no consideration of how the machines will be arranged within the cell, how the materials move in a cell, and how much machine investment is required to process the parts assigned to that cell. There are few studies that consider the GT layout problem such as Vakharia and Wemmerlöv (1990), Dahel (1995), Verma and Ding (1995), Akturk and Balkose (1996), and BazarganLari and Kaebernick (1996). Therefore, the configuration and the performance measures of the cell must be considered throughout the design process in order to provide information for the selection of the best part-family and machine-cell formation and spatial arrangements of machines in each cell.

Every firm should make profit to stay in the market. In most of the studies, one or more of the manufacturing costs; variable production cost, setup cost, inter-cell and intra-cell material handling costs, or additional machine investment cost, are tried to be minimized while solving the PFMCF problem. The cost minimization objective is an important one when we consider the overall system performance. Although it causes a kind of dependency between cells when the performance of the individual cells is of concern. For example, a cell that is loosing money is dependent on another cell making profit. Therefore, we employ a holonistic approach to solve CMS design problem by considering both the performance of individual cells and the overall system. A holonistic manufacturing system (HMS) is a system of holons that can cooperate to achieve a common goal or objective. 'Holon' is an identifiable part of a system that is made up of subordinate parts and has a unique identity. They are autonomous and cooperative building blocks of the system. The holonic organization enables the construction of very complex systems that are efficient in the use of resources, highly resilient to external and internal disturbances and adaptable to changes as discussed in Höpf (1994). There are several similarities between HMS and CMS. CMS design starts with forming cells which are similar to holons in HMS. In HMS, the holons have tasks to perform. In CMS, part families and machine groups are formed to determine the tasks of cells. The main 
difference between the traditional cellular manufacturing systems and HMS is the autonomy of the entities in HMS. Autonomy, which is the capability of an entity to create and control its own plans and strategies, is mostly not found in cells in CMS. We introduce the autonomy concept to the CMS design problem to form selfsufficient cells.

The remainder of the paper is organized as follows. In section 2, we define the scope of the study with underlying assumptions and state a mathematical formulation of the problem. In section 3, we present the proposed solution procedure, which is applied in an example problem in section 4. The results of the experimental design to test the efficiency of the algorithm are discussed in section 5. Finally, some concluding remarks are provided in the last section.

\section{Problem statement}

In this study, our aim is to solve the PFMCF and layout problems simultaneously using a holonistic approach to maximize profit of not only the overall system but also individual cells. While forming cells and determining their withincell layouts, several important manufacturing issues such as production volumes, processing times, operation sequences, alternative routings and machine utilization levels are incorporated into the design problem. The profit maximization objective is inspired from an application to a state-owned manufacturing company in Turkey. The company has divided some part of its factory into small holons in order to implement HMS. Since the company is being privatized, these holons are sold to individuals to form small enterprises. These enterprises produce parts for the company and sell them to the company. They also have the ability to produce for other firms. The main characteristics of holons, which are the cooperativeness of entities to achieve common goals and the autonomy of them to create and control their own plans and strategies, seem to exist in these enterprises. But, most of these enterprises face economical problems that limit their autonomy. They cannot make enough profit due to low utilization levels and high number of inter-cell movements. The enterprises should make profit to achieve self-sufficiency and to stay in the market. Therefore, in the proposed algorithm, the efficiency of the individual cells is tried to be achieved with the use of minimum profit level for cells. The profit of the system, which is affected by the profit of individual holons, is also important for the holons since they cooperate to achieve a common goal. So, profit maximization objective together with the low profit level constraints are used in this model.

The number of parts and machine types are assumed to be known a priori. Each part has a fixed demand, alternative routings, and predetermined processing times on each route. Processing times, together with the part volume, are used in determining the number of machines required of each type. The operation sequences of parts are important in determining the within-cell layout. In this study, the machines in the cells are located next to each other in series to form a flow-line manufacturing cell. If there is more than one machine of the same type assigned to a cell, the duplicate machines are assumed to be located in parallel. The raw material, production, material handling and additional machine investment costs, and selling prices of the parts are assumed to be known. These monetary terms will be included in the profit maximization objective. Under these assumptions, part families and routings of parts, machine groups, part 
assignments to cells, machine assignments to cells in terms of quantities and the location of machines in each cell will be determined.

We propose a mixed integer programming (MIP) model to maximize the profit under cell size, low utilization and low profit level constraints while determining the layout, part assignments, routing selections and machine assignments.

The parameters of the problem are as follows:

$p$ number of cells,

$n$ number of parts,

$M$ set of all machine types,

$R_{i}$ number of routes for part $i$,

$D_{i}$ demand for part $i$

$Y_{i r k l}$ 0-1 binary indicator which is equal to 1 if part $i$ should be processed by machine type $k$ immediately before machine type $l$ in its $r$ th route,

$h_{i}$ unit material handling cost of part $i$ within a cell,

$c_{i r k}$ unit production cost of part $i$ using route $r$ on machine $k$ per unit time,

$t_{i r k}$ unit processing time of part $i$ using route $r$ on machine $k$,

$S P_{i} \quad$ selling price of part $i$,

$R M_{i}$ raw material cost of part $i$,

$L P_{j}$ lower limit for the profit of cell $j$,

$A_{k}$ available unit capacity of machine type $k$,

$M A_{k}$ available number of machine type $k$,

$M C_{k}$ additional machine investment cost of machine $k$,

$C S_{j}$ an upper limit on the number of machines assigned to cell $j$,

$U$ a very large constant,

$M_{i r}$ set of machines in the $r$ th route of part type $i$,

$\gamma_{k j}$ lower limit for the utilization levels of machine type $k$ in cell $j$.

The decision variables are the following:

$X_{i r j}$ 0-1 binary variable which is equal to 1 if part $i$ is allocated to cell $j$ using the $r$ th route,

$m_{k j} \quad 0-1$ binary variable which is equal to 1 if machine $k$ is allocated to cell $j$,

$m l_{k j}$ location of machine $k$ in cell $j$,

$S_{k l j}^{+}, S_{k l j}^{-}$number of skippings and backtrackings between machines $k$ and $l$ in cell $j$,

$\alpha_{k l j} \quad 0-1$ binary variable which is equal to 1 if machine $l$ is placed after (not necessarily immediately) machine $k$ in cell $j$,

$\delta_{i r j k l} 0-1$ binary variable which is equal to 1 if part $i$ uses route $r$ and allocated to cell $j$, where machine $k$ is not located immediately before machine $l$,

$\lambda_{j} 0-1$ binary variable which is equal to 1 if cell $j$ is opened,

$N_{k j}$ number of type $k$ machines assigned to cell $j$,

$M N_{k}$ number of additional machines of type $k$ needed.

A MIP formulation of the problem is as follows:

- Objective function 


$$
\begin{aligned}
\operatorname{Maximize} & {\left[\sum_{i=1}^{n}\left(S P_{i}-R M_{i}\right) \cdot D_{i} \cdot\left(\sum_{r \in R_{i}} \sum_{j=1}^{p} X_{i r j}\right)\right] } \\
& -\left[\sum_{j=1}^{p} \sum_{i=1}^{n} \sum_{r \in R_{i}} \sum_{k \in M_{i r}} \sum_{l \in M_{i r}} D_{i} \cdot h_{i} \cdot \delta_{i r j k l}\right] \\
& -\left[\sum_{j=1}^{p} \sum_{i=1}^{n} \sum_{r \in R_{i}} \sum_{k \in M_{i r}} D_{i} \cdot c_{i r k} \cdot t_{i r k} \cdot X_{i r j}\right] \\
& -\left[\sum_{k \in M} M C_{k} \cdot M N_{k}\right] .
\end{aligned}
$$

- Part and machine allocation, and routing selection constraints

$$
\begin{gathered}
\sum_{j=1}^{p} \sum_{r \in R_{i}} X_{i r j}=1 \quad \forall i \\
\sum_{r \in R_{i}} X_{i r j} \leq \lambda_{j} \quad \forall i, j \\
X_{i r j} \leq m_{k j} \quad \forall i, r, j, k \in M_{i r} \\
m_{k j} \leq \lambda_{j} \quad \forall k, j .
\end{gathered}
$$

- Layout related constraints

$$
\begin{gathered}
m_{k j} \geq \frac{m l_{k j}}{U} \quad \forall k, j \\
m_{k j} \leq m l_{k j} \quad \forall k, j \\
m l_{k j}-m l_{l j}+U \alpha_{k l j} \geq m_{k j}+m_{l j}-1 \quad \forall k, l, j \\
m l_{l j}-m l_{k j}+U\left(1-\alpha_{k l j}\right) \geq m_{k j}+m_{l j}-1 \quad \forall k, l, j \\
S_{k l j}^{+}-S_{k l j}^{-}=m l_{l j}-m l_{k j} \quad \forall k, l, j \\
\left(S_{i r j k l}^{+}+\left(1-X_{i r j}\right)-\frac{S_{k l j}-1}{2 U}-\frac{S_{k l j}^{-}}{U}\right) Y_{i r k l} \geq 0 \quad \forall i, r, j, k, l .
\end{gathered}
$$

- Low profit level constraint

$$
\begin{aligned}
& \sum_{i=1}^{n} \sum_{r \in R_{i}} D_{i} \cdot\left(S P_{i}-R M_{i}\right) \cdot X_{i r j}-\sum_{i=1}^{n} \sum_{r \in R_{i}} \sum_{k \in M_{i r}} \sum_{l \in M_{i r}} D_{i} \cdot h_{i} \cdot \delta_{i r j k l} \\
& \quad-\sum_{i=1}^{n} \sum_{r \in R_{i}} \sum_{k \in M_{i r}} D_{i} \cdot c_{i r k} \cdot t_{i r k} \cdot X_{i r j} \geq L P_{j} \quad \forall j .
\end{aligned}
$$

- Low utilization level constraint

$$
\sum_{i=1}^{n} \sum_{r \in R_{i}} t_{i r k} D_{i} X_{i r j} \geq \gamma_{k j} \quad \forall k, j .
$$


- Machine capacity constraints (also determines the additional machines needed)

$$
\begin{aligned}
\sum_{i=1}^{n} \sum_{r \in R_{i}} t_{i r k} D_{i} X_{i r j} \leq N_{k j} A_{k} & \forall k, j \\
\sum_{j=1}^{p} N_{k j}-M N_{k} \leq M A_{k} & \forall k \\
m_{k j} \geq N_{k j} / U & \forall k, j \\
m_{k j} \leq N_{k j} & \forall k, j
\end{aligned}
$$

- Cell Size constraint

$$
\sum_{k \in M} N_{k j} \leq C S_{j} \quad \forall j
$$

- Non-negativity and integrality constraints

$$
X_{i r j}, \lambda_{j}, m_{k j}, \alpha_{k l j}, \delta_{i r j k l}=0,1
$$

and

$$
m l_{k j}, N_{k j}, M N_{k} \text { non-negative integers } \forall i, r, j, k, l .
$$

The overall objective is to maximize the total profit. The first item in the objective function is the difference between the revenue and the raw material cost. This term does not change by the cell that the part is assigned or the route selected for the part. The material handling and variable production costs, denoted as the manufacturing cost, are the second and third terms in the objective function, respectively. The material handling cost is incurred to the parts making an intra-cell movement. The variable production cost changes according to the route selected. Since additional machines may be needed to form completely independent cells, the additional machine investment cost, which is the fourth term in the objective function, should be as small as possible.

The first set of constraints satisfy the assignment of each part to only one cell and selection of only one route among alternative routing capabilities. The second and third set of constraints ensure that a part can be assigned to a cell if it is opened and contain all of the machines needed to process the part. Constraints 4, 5 and 6 provide that if a machine is allocated to a cell then the location of the machines should be greater than one. Constraints 7 and 8 ensure the assignment of machines to different locations in a cell such that two machines cannot occupy the same place in a cell. Constraint 9 determines the number of skippings and backtrackings between the machines in a cell. In constraint 10, a material handling cost is not incurred when the two consecutive machines in the operation sequence are next to each other in the forward flow direction. On the other hand, a material handling cost is incurred for the backtrackings and the skippings which are determined according to the order of machines in the cell and the operation sequences of parts. Skippings and backtrackings are given equal weights in this problem, since they are both performed by the same type of material handling equipment. The cells should make at least a predetermined amount of profit which is satisfied by constraint 11 . This comes from the holonistic view to form autonomous, self-sufficient cells. Constraint 12 allows the 
decision makers to specify a lower limit on the desired machine utilization in a cell. Constraint 13 determines the number of machines of each type required in each cell. Constraint 14 finds the number of additional machines needed to calculate the additional machine investment cost. Constraints 15 and 16 provide the assignment of a machine to a cell if it is needed in that cell. There are several physical constraints, such as two machines cannot occupy the same place in a cell as discussed above. Another one could be space limitations. Therefore, the size of a cell is often determined by the floor area of the factory which sets an upper limit on the number of machines a cell can have. The size of the cell should be a reasonable one to make the controlling, scheduling, and planning activities easier in the cell, which is ensured by constraint 17.

\section{Algorithm}

A local search heuristic is proposed to solve the problem in a reasonable computation time to form completely independent cells. The proposed algorithm has three main stages. The first two stages are used to find a solution to the main problem (MP). Since the inter-cell movements are also important for reducing the additional machine investment cost, the assumption of forming completely independent cells can be relaxed in the algorithm. In the third stage, inter-cell movements are introduced to decrease additional machine investment cost, hence improve the objective function value.

\section{Stage 1: Finding an initial solution}

In the proposed algorithm, the first stage is the construction of an initial solution. In this stage, the within-cell layout and low utilization level constraints are relaxed, and the layout related constraints, non-negativity and integrality constraints of MP (constraints 5-10, and 18) are replaced with the following constraints to form a new subproblem.

$$
\begin{array}{cc}
m_{k j}-\sum_{l \in M} \beta_{l k j} \geq 0 & \forall k, j \\
m_{k j}-\sum_{l \in M} \beta_{k l j} \geq 0 & \forall k, j \\
m_{k j}-\sum_{l \in M} \beta_{k l j}-\sum_{l \in M} \beta_{l k j} \leq 0 & \forall k, j \\
\left(\delta_{i r k l l}-X_{i r j}+\beta_{k l j}\right) Y_{i r k l} \geq 0 & \forall i, r, j, k, l \\
X_{i r j}, \lambda_{j}, m_{k j}, \beta_{k l j}=0,1 \text { and } N_{k j} \text { are integers } & \forall i, r, j, k, l .
\end{array}
$$

where $\beta_{k l j}$ is equal to 1 if machine $k$ is placed just before machine $l$ in cell $j$ and 0 otherwise.

The constraints of the subproblem are 1-4, 11-17, and 19-23. The disadvantage of the new layout related constraints is that the locations of machines in the cell cannot be determined exactly. We can only find the machines next to each other, hence the final layout can be infeasible. The same type of machine can be duplicated at the beginning and at the end of a group of machines in a cell without incurring an additional machine investment cost. The computational time needed to solve subproblem is significantly less than the time needed to solve the MP. In the main problem, $m l_{k j}$ 's should be defined as integers and $\delta_{i r j k l}$ 's as binary variables. MP 
has additional $(3 m+2) \times m \times p$ constraints, $m \times p$ integer and $n \times p \times \bar{R} \times$ $(\overline{N O P}-1)$ binary variables. $\bar{R}$ is the average number of routes for parts and $\overline{N O P}$ is the average number of operations in a part's sequence. After the layout constraints are replaced, the low utilization level constraint is also relaxed to reduce the computation time even further. The relaxed subproblem (RSP) can be solved optimally in a reasonable time for small problems, although it may or may not be feasible for MP, since the layout and low utilization level constraints are relaxed. If the solution is infeasible for MP, then stage 2 is used to find a feasible solution. The steps of stage 1 are as follows:

1.1 Form R SP and solve it by using the CPLEX software to find an initial solution. The current solution $(C S)$ is the solution found by solving RSP.

1.2 Determine the layout found by RSP. Calculate the utilization level of each machine in each cell by $U t i l_{k j}=\sum_{i} \sum_{r \in R_{i}} t_{i r k} D_{i} X_{i r j}$. If either the layout is infeasible or low utilization level constraint is not satisfied then the solution is infeasible for MP, go to stage 2. Else, go to stage 3 if any additional machine investment is required to form completely independent cells.

\section{Stage 2: Formation of independent cells}

In the second stage, alternative solutions are constructed by assigning parts to other cells and/or selecting alternative routes for parts to find a feasible solution to the MP. The promising alternatives are found by calculating the change in the objective function value and one promising alternative is selected randomly to perturb the solution. The procedure continues by searching the neighborhood of the current solution until a stopping criterion is reached. The criteria can be finding either a feasible solution or reaching to a maximum limit on step size, or having no further improvement in the objective value. In the next step, the feasible solution is tried to be improved by continuing local search. These steps are performed for a number of iterations to reduce the effect of randomness in selecting the alternatives. While performing the local search, the feasible solution giving the best objective value is kept as an incumbent solution to the proposed mathematical model. The steps of stage 2 are as follows:

2.1 If the layout of cells are found to be infeasible for MP in stage 1, determine alternative layouts for each cell.

2.2 Take an initial layout for each cell. Update $C S$ in terms of layout.

2.3 $C S^{\prime}=C S$. For $C S^{\prime}$, calculate utilization levels of machines in each cell and total profit of each cell $\left(T P_{j}\right)$, such that $T P_{j}=S P R M_{j}-V P C_{j}-M H C_{j}$, where

$$
\begin{aligned}
M H C_{j} & =\sum_{i} \sum_{r \in R_{i}} \sum_{k \in M R_{i r}} \sum_{l \in M R_{i r}} h_{i} \cdot D_{i} \cdot\left(1-\beta_{k l j}\right) \cdot X_{i r j} \\
V P C_{j} & =\sum_{i} \sum_{r \in R_{i}} \sum_{k \in M R_{i r}} c_{i r k} \cdot t_{i r k} \cdot D_{i} \cdot X_{i r j} \\
S P R M_{j} & =\sum_{i} \sum_{r \in R_{i}}\left(S P_{i}-R M_{i}\right) \cdot D_{i} \cdot X_{i r j} .
\end{aligned}
$$

If either low profit level constraint or low utilization level constraint is not satisfied go to step 2.4, else go to step 2.5 .

2.4 Search the neighborhood of $C S^{\prime}$ to find a feasible solution. 
2.4.1 Find the alternative parts that can be assigned to other cells and the corresponding promising cells to which the alternative parts can be assigned. If machine $k$ in cell $j$ violates the low utilization level constraint, the parts using this machine in cell $j$ and the parts using the machine of the same type in other cells are candidates that can be assigned to other cells. A promising cell, to which an alternative part can be assigned, contains a certain percentage of machines in a part's routing denoted by $\theta$, and satisfies the following condition $\left(N O P_{i}-\#\right.$ machines needed $) / N O P_{i} \geq \theta$.

2.4.2 For each alternative, determine the within-cell layout and calculate the corresponding cost terms in cells. When part $i$ using route $r$ in cell $j$ is assigned to cell $j j$ and produced with its $r r$ th route, the cost terms change as follows:

$$
\begin{aligned}
\text { New } M H C_{j^{\prime}}= & \sum_{i} \sum_{r \in R_{i}} \sum_{k \in M R_{i r}} \sum_{l \in M R_{i r}} h_{i} \cdot D_{i} \cdot\left(1-\beta_{k l j^{\prime}}\right) \cdot X_{i r j^{\prime}}, \\
& \quad \text { or } j^{\prime}=j, j j \\
\text { New } V P C_{j}= & \text { Old } V P C_{j}-\sum_{k \in M R_{i, r}} c_{i r k} \cdot t_{i r k} \cdot D_{i} \\
\text { New } V P C_{j j}= & \text { Old } V P C_{j j}+\sum_{k \in M R_{i, r}} c_{i, r r, k} \cdot t_{i, r r, k} \cdot D_{i} \\
\text { New } S P R M_{j}= & \text { Old } S P R M_{j}-\left(S P_{i}-R M_{i}\right) \cdot D_{i} \\
\text { New } S P R M_{j j}= & \text { Old } S P R M_{j j}+\left(S P_{i}-R M_{i}\right) \cdot D_{i} \\
\text { New } T P_{j^{\prime}}= & \text { New } S P R M_{j^{\prime}}-\mathrm{New} V P C_{j^{\prime}}-\text { New } M H C_{j^{\prime}}, \\
& \quad \text { for } j^{\prime}=j, j j .
\end{aligned}
$$

If the cells $j$ and $j j$ do not violate the low profit level constraints, the change in the objective function value for the alternative $(i, r r, j j)$ is calculated as follows:

$$
\begin{aligned}
\Delta O b j_{i, r r, j j}= & \text { New } T P_{j}-\text { Old } T P_{j}+\text { New } T P_{j j}-\text { Old } T P_{j j} \\
& +M I C 1-M I C 2,
\end{aligned}
$$

where $M I C 1$ is the money spend for the machines which are not needed anymore and $M I C 2$ is the total cost needed to buy the new required machines.

2.4.3 Form a restricted candidate list (RCL). Every alternative $\left(i^{\prime}, r r^{\prime}, j j^{\prime}\right)$ is added to the RCL if it satisfies the following condition of $\left(\max _{i, r r, j j}\left\{\Delta O b j_{i, r r, j j}\right\}-\Delta O b j_{i^{\prime}, r r^{\prime}, j j^{\prime}}\right) \leq(\mu \cdot O b j)$, where $\mu$ is a predefined parameter to select the promising alternatives. Select one alternative from the list randomly and update $C S^{\prime}$. According to the selected alternative, $\left(i^{*}, r r^{*}, j j^{*}\right)$, part $i^{*}$ is produced in cell $j j^{*}$ with its $r r^{*}$ th route, and the objective value is updated as New $O b j=$ Old $O b j+\Delta O b j_{i^{*}, r r^{*}, j j^{*}}$.

2.4.4 Repeat steps 2.4.1-2.4.3 until a stopping criterion is reached. The stopping criteria are finding a feasible solution or reaching to the maximum step size. 
2.5 Try to improve the feasible solution by searching the neighborhood of the current solution. This step is similar to the previous step except that determination of alternative parts and the stopping criteria differs. All parts are candidates that can be assigned to other cells. The stopping criteria are having no further improvement in the objective function value or reaching to the maximum step size.

2.6 Repeat steps 2.3-2.5 for a fixed number of iterations.

2.7 Repeat steps 2.2-2.6 until all alternative layouts are evaluated.

2.8 Best feasible solution is reported as the final solution. If any additional machine investment is required to form completely independent cells, then go to stage 3 .

\section{Stage 3: Allowing inter-cell movements}

At the end of second stage, an additional investment might have been made for some machines to form completely independent cells. Decision maker may allow some inter-cell movements to reduce the additional machine investment cost. Intercell movements can also increase the overall profit by giving a better material flow, although they complicate the scheduling within cells. While inter-cell movements are allowed, the objective of forming profitable cells is not violated. An important distinction from a typical minimization of inter-cell movements objective is the allocations of profit and manufacturing costs between the interacted cells. While calculating the material handling cost, the intra-cell movement costs are incurred to the cell in which the intra-cell movement takes place. The inter-cell movement costs are incurred to the cell to which the part is assigned. The variable production cost for each operation is incurred to the cell in which this operation is performed. The difference between the revenue and the raw material cost, denoted as gain, is divided between two cells. The gain for each cell is proportional with the variable production cost incurred for the part in that cell. At the end of this stage, cells having interaction with each other via inter-cell moves are formed. The steps of stage 3 are as follows.

3.1 Take the best solution obtained at the end of stage 2 as an initial solution.

3.2 Determine the alternative parts that can make an inter-cell movement and their corresponding cells. Parts, which are not making an inter-cell movement but require an additional machine investment, are allowed to make an inter-cell movement. Parts can be assigned to their promising cells as in step 2.4.1. The operations that cannot be performed in the promising cell are performed in another cell to which alternative part can make an inter-cell movement.

3.3 When part $i$, which was using its $r$ th route in cell $j$, is assigned to cell $j j$, and allowed to make an inter-cell movement to cell $j j j$ in order to be processed with its $r r$ th route, the cost terms change as follows:

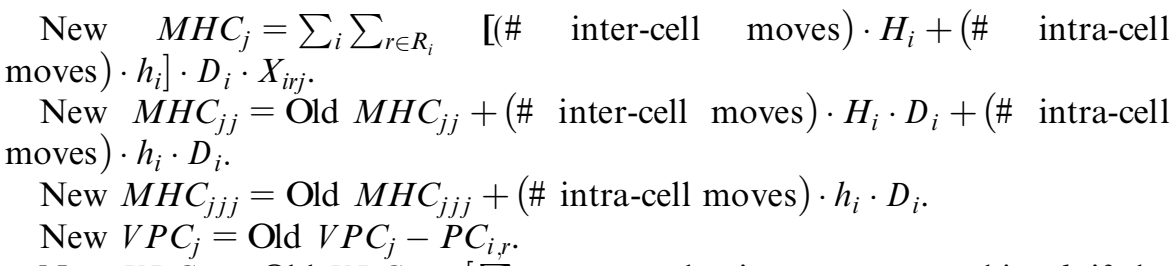

New $V P C_{j j}=$ Old $V P C_{j j}+\left[\sum_{k \in M R_{i, r}}\right.$ production cost on machine $k$ if the operation on machine $k$ is performed in cell $j j$, denoted as $\left.W I P_{i, r r, j}\right]$.

New $V P C_{j j j}=$ Old $V P C_{j j}+\left[\sum_{k \in M R_{i, r}}\right.$ production cost on machine $k$ if the 
operation on machine $k$ is performed in cell $j j j]$.

New $S P R M_{j}=$ Old $S P R M_{j}-T S P_{i}$.

New $S P R M_{j j}=\operatorname{Old} S P R M_{j j}+\frac{W I P_{i, r r j j}}{P C_{i, r r}} \cdot T S P_{i}$.

New $S P R M_{j j j}=$ Old $S P R M_{j j j}+\frac{P C_{i, r r}-W I P_{i, r r, j j}}{P C_{i, r r}} \cdot T S P_{i}$.

$T P_{j^{\prime}}=$ New $S P R M_{j^{\prime}}-$ New $V P C_{j^{\prime}}-$ New $M H C_{j^{\prime}}$, for $j^{\prime}=j, j j, j j j$.

If low profit level constraint is satisfied by cells $j, j j$ and $j j j$, the change in objective function value for alternative $(i, r r, j j, j j j)$ can be calculated as follows:

$$
\begin{aligned}
\Delta O b j_{i, r, j j, j j j}= & \text { New } T P_{j}-\text { Old } T P_{j}+\text { New } T P_{j j}-\text { Old } T P_{j j} \\
& + \text { New } T P_{j j j}-\text { Old } T P_{j j j}+M I C 1 .
\end{aligned}
$$

3.4 Form the RCL as it is explained in step 2.4.3. If no alternative can be found, go to step 3.5. Otherwise, select one of the alternatives from the candidate list randomly. According to the selected alternative, say $\left(i^{*}, r r^{*}, j j^{*}, j j j^{*}\right)$, the solution is updated. In the current solution, part $i^{*}$ makes inter-cell movement between cells $j j^{*}$ and $j j j^{*}$ to be processed with its $r r^{*}$ th route. Return back to step 3.2.

3.5 Repeat steps 3.1-3.4 for a fixed number of iterations.

3.6 While performing all these steps, the solution giving the best objective value is kept as the incumbent solution.

3.7 Form a from-to-chart by using the number of inter-cell moves and production volumes. The total number of inter-cell moves from cell $j j$ to $j j j$ is equal to $\sum_{i=1}^{n}(\#$ inter-cell moves from cell $j j$ to $j j j) \cdot D_{i}$. Note that the from-to-chart is not symmetric. Form a cost chart considering the inter-cell material handling costs between cells. Find the area of each cell by considering the machines assigned to them. The machines of different types are located in series in a flow line, whereas the duplicate machines are located in parallel. If there is not enough space in the factory, the same type of machines can be located next to each other in series. Find the spatial arrangement of cells in the factory by using the CRAFT algorithm.

\section{Numerical example}

A numerical example is provided to clarify the basic steps of the algorithm. The routing, processing time, production cost, inter-cell and intra-cell material handling costs, the difference between selling price and raw material cost, and demand of each part can be seen in table 1. Furthermore, the additional investment cost and the available number for each machine type are given in table 2 . The minimum profit level for each cell is taken as 746 .

As discussed earlier, the first stage of the algorithm is used to find an initial solution. The relaxed subproblem is solved by CPLEX. The layout of each cell, the parts $(i)$ assigned to the cells and the routings selected for the parts $(r)$ can be seen in table 3, and the objective function value is 10392 . Although the utilization level constraint is satisfied by all machines, the layout of cells are infeasible for MP. 


\begin{tabular}{|c|c|c|c|c|c|c|c|c|}
\hline$i$ & $R_{i}$ & $\mathrm{k},\left(t_{\text {irk }}, c_{\text {irk }}\right)$ & & & $H_{i}$ & $h_{i}$ & $S P_{i}-R M_{i}$ & $D_{i}$ \\
\hline 1 & 1 & $2(1,3.20)$ & $4(5,2.77)$ & & 6.38 & 5.11 & 21.31 & 154 \\
\hline 1 & 2 & $1(1,3.84)$ & $5(2,3.32)$ & & 6.38 & 5.11 & 21.31 & 154 \\
\hline 2 & 1 & $6(3,2.68)$ & $2(3,3.41)$ & & 6.83 & 5.47 & 22.80 & 122 \\
\hline 2 & 2 & $2(5,3.21)$ & $1(2,4.09)$ & & 6.83 & 5.47 & 22.80 & 122 \\
\hline 3 & 1 & $3(4,2.73)$ & $4(2,3.08)$ & $5(4,2.53)$ & 6.79 & 5.44 & 33.99 & 170 \\
\hline 3 & 2 & $3(4,3.27)$ & $6(4,3.70)$ & $5(3,3.04)$ & 6.79 & 5.44 & 33.99 & 170 \\
\hline 4 & 1 & $3(3,2.80)$ & $4(2,3.32)$ & $1(1,2.90)$ & 4.47 & 3.59 & 22.42 & 109 \\
\hline 4 & 2 & $2(5,3.36)$ & $1(5,3.98)$ & $6(4,3.48)$ & 4.47 & 3.59 & 22.42 & 109 \\
\hline 5 & 1 & $1(2,3.15)$ & $4(3,2.86)$ & & 5.58 & 4.46 & 18.61 & 151 \\
\hline 5 & 2 & $3(5,3.78)$ & $4(3,3.44)$ & & 5.58 & 4.46 & 18.61 & 151 \\
\hline 6 & 1 & $2(2,2.83)$ & $4(2,3.40)$ & & 4.67 & 3.74 & 15.60 & 134 \\
\hline 7 & 1 & $6(4,2.62)$ & $4(2,2.98)$ & $1(4,2.81)$ & 6.92 & 5.54 & 34.63 & 158 \\
\hline 7 & 2 & $1(4,3.15)$ & $4(3,3.57)$ & $3(2,3.38)$ & 6.92 & 5.54 & 34.63 & 158 \\
\hline 8 & 1 & $4(1,3.21)$ & $6(2,2.54)$ & & 3.10 & 2.48 & 10.36 & 121 \\
\hline 8 & 2 & $5(4,3.86)$ & $6(4,3.04)$ & & 3.10 & 2.48 & 10.36 & 121 \\
\hline 9 & 1 & $4(5,2.99)$ & $3(5,3.16)$ & & 11.52 & 9.22 & 38.42 & 137 \\
\hline 10 & 1 & $3(3,2.98)$ & $4(3,3.24)$ & & 6.99 & 5.59 & 23.31 & 141 \\
\hline 10 & 2 & $5(2,3.58)$ & $4(5,3.88)$ & & 6.99 & 5.59 & 23.31 & 141 \\
\hline 11 & 1 & $6(5,3.36)$ & $3(5,2.96)$ & & 11.84 & 9.48 & 39.50 & 187 \\
\hline 11 & 2 & $5(4,4.03)$ & $4(1,3.55)$ & & 11.84 & 9.48 & 39.50 & 187 \\
\hline 12 & 1 & $3(2,2.70)$ & $6(3,2.56)$ & $5(1,2.94)$ & 4.00 & 3.20 & 20.00 & 168 \\
\hline 13 & 1 & $2(4,2.98)$ & $6(2,3.02)$ & $4(5,3.04)$ & 8.27 & 6.62 & 41.41 & 107 \\
\hline 13 & 2 & $5(3,3.57)$ & $4(1,3.63)$ & $2(3,3.64)$ & 8.27 & 6.62 & 41.41 & 107 \\
\hline 14 & 1 & $6(1,2.98)$ & $1(1,3.15)$ & & 2.29 & 1.84 & 7.66 & 161 \\
\hline 14 & 2 & $1(4,3.57)$ & $3(1,3.78)$ & & 2.29 & 1.84 & 7.66 & 161 \\
\hline 15 & 1 & $6(1,2.51)$ & $5(1,2.65)$ & & 1.93 & 1.55 & 6.45 & 149 \\
\hline 15 & 2 & $5(1,3.02)$ & $2(3,3.18)$ & & 1.93 & 1.55 & 6.45 & 149 \\
\hline 16 & 1 & $1(2,3.03)$ & $5(1,3.21)$ & $2(2,2.61)$ & 3.61 & 2.89 & 18.10 & 110 \\
\hline 17 & 1 & $2(3,2.70)$ & $5(2,3.36)$ & $6(4,2.54)$ & 6.25 & 5.00 & 31.26 & 175 \\
\hline 18 & 1 & $6(4,2.52)$ & $2(3,3.16)$ & $5(5,2.71)$ & 8.27 & 6.62 & 41.37 & 154 \\
\hline 18 & 2 & $2(1,3.02)$ & $4(5,3.79)$ & $6(1,3.25)$ & 8.27 & 6.62 & 41.37 & 154 \\
\hline 19 & 1 & $6(3,2.61)$ & $5(4,2.90)$ & $2(3,3.01)$ & 7.11 & 5.69 & 35.60 & 123 \\
\hline 19 & 2 & $4(3,3.13)$ & $6(1,3.48)$ & $2(3,3.61)$ & 7.11 & 5.69 & 35.60 & 123 \\
\hline 20 & 1 & $5(3,3.48)$ & $6(4,2.55)$ & & 7.74 & 6.19 & 25.81 & 174 \\
\hline
\end{tabular}

Table 1. Parameters for the example.

\begin{tabular}{lcccccc}
\hline Machines & 1 & 2 & 3 & 4 & 5 & 6 \\
\hline MC & 1529 & 1401 & 1532 & 1568 & 1572 & 1486 \\
MA & 1 & 1 & 2 & 3 & 1 & 3 \\
\hline
\end{tabular}

Table 2. Additional investment cost and the available number of machines.

\begin{tabular}{|c|c|c|}
\hline Cell & Layout & Parts $(i, r)$ \\
\hline 1 & $6156-4234$ & $(1,2)(3,1)(4,1)(10,1)(13,2)(14,1)(16,1)(17,1)$ \\
\hline 2 & $3143-656$ & $(5,1)(7,2)(9,1)(11,2)(12,1)(15,1)(20,1)$ \\
\hline 3 & 6246 & $(2,1)(6,1)(8,1)(18,2)(19,2)$ \\
\hline
\end{tabular}

Table 3. Solution of the relaxed problem. 
Since the solution is infeasible, we proceed to the second stage to find a feasible solution to the main problem.

The second stage begins with finding alternative layouts for each cell. A limit, 3, on the maximum number of alternative layouts for a cell is used in this example to control the size of the search space. The alternative layouts for each cell can be seen in table 4 .

The initial layouts for cells 1,2 and 3 are taken as $(1-5-6-2-3-4),(1-4-3-6-5)$ and (6-2-4), respectively, from the set of alternatives. For the selected initial layouts, total profit of cells 1, 2 and 3 are 3222, 4960 and 3153, respectively. The minimum profit level constraint is satisfied and additional machine investment cost is 4502 . Although the objective function value of the relaxed problem was 10392 , the objective function value of the initial feasible solution is 6830 due to the increase in material handling cost. At this point, both the low utilization and low profit level constraints are satisfied for the initial solution. Since the current solution is feasible for MP, we have to search the neighborhood of the current solution in order to see whether the solution can be improved or not.

All the parts are denoted as alternative parts since they can be assigned to their promising cells. $\theta$ is taken as 0.3 in this example. The change in objective function value $(\Delta O b j)$ is calculated for all alternatives. Since $\Delta O b j$ values for some alternatives are greater than 0 , a restricted candidate list is formed. $\mu$, which determines the size of the RCL, is taken as 0.1 . Furthermore, $O b j$ is 6830 and maximum of $\triangle O b j$ values is 1319 . Alternatives $(2,1,1)$, for which $\Delta O b j=1319$, and $(11,2,4)$, for which $\Delta O b j=699$, are the only alternatives that can be included in the R CL. Suppose we select the alternative $(2,1,1)$ from the R CL. Now, part 2 using route 1 is produced in cell 1 and the new objective value becomes 8149 . For the current solution, a new set of alternatives are determined and their $\Delta O b j$ values are calculated. Since $\Delta O b j$ for all alternatives are smaller than zero, we cannot improve the current objective value.

The first iteration ends with the stopping criterion of having no alternative left. This search is repeated for a number of iterations until all alternative layouts are evaluated. At the end of this stage, the final solution along with the PFMCF and layout of cells can be seen in table 5. Additional machine investment cost is 6074 and Obj is equal to 9314. The material flow in each cell for the final solution can be seen in figure 1, where the numbers on each arc correspond to part numbers, whereas the

\begin{tabular}{|c|c|c|c|c|c|}
\hline Cell & Layout & Cell & Layout & Cell & Layout \\
\hline 1 & $\begin{array}{llllll}6 & 1 & 5 & 4 & 2 & 3 \\
1 & 5 & 6 & 2 & 3 & 4 \\
1 & 5 & 6 & 4 & 2 & 3\end{array}$ & 2 & $\begin{array}{lllll}3 & 1 & 4 & 6 & 5 \\
1 & 4 & 3 & 5 & 6 \\
1 & 4 & 3 & 6 & 5\end{array}$ & 3 & $\begin{array}{ll}624 \\
246\end{array}$ \\
\hline
\end{tabular}

Table 4. Alternative layouts for cells.

\begin{tabular}{lll}
\hline Cell & Layout & \multicolumn{1}{c}{ Parts $(i, r)$} \\
\hline 1 & 156234 & $(1,2)(3,1)(4,1)(10,1)(14,1)(16,1)(17,1)(20,1)$ \\
2 & 14365 & $(5,1)(7,2)(9,1)(12,1)(15,1)$ \\
3 & 5462 & $(2,1)(6,1)(8,1)(11,2)(13,2)(18,2)(19,2)$ \\
\hline
\end{tabular}

Table 5. Final solution without an intercell movement. 

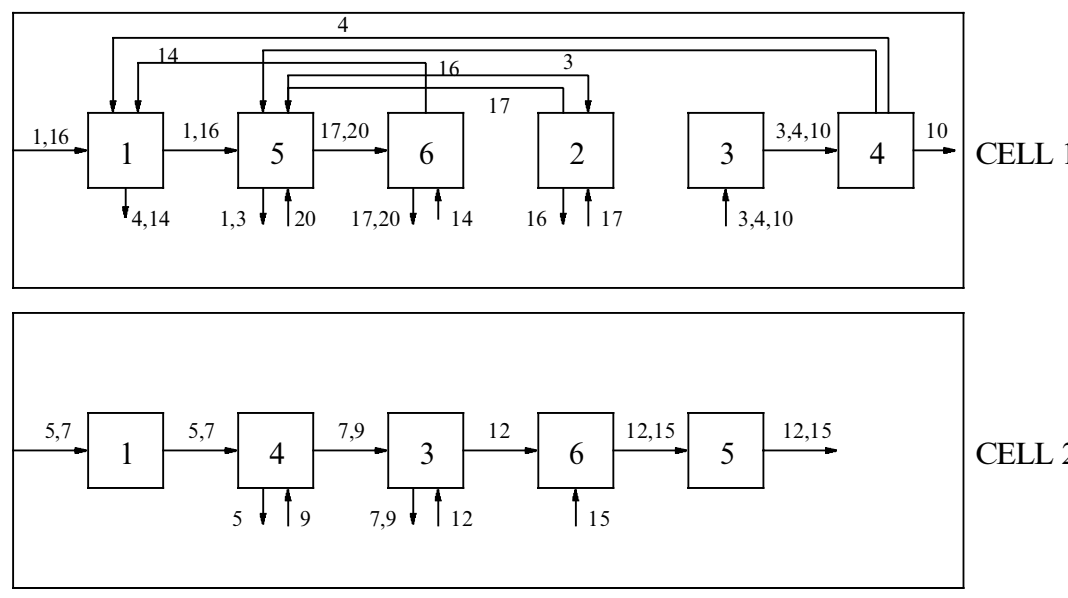

CELL 2

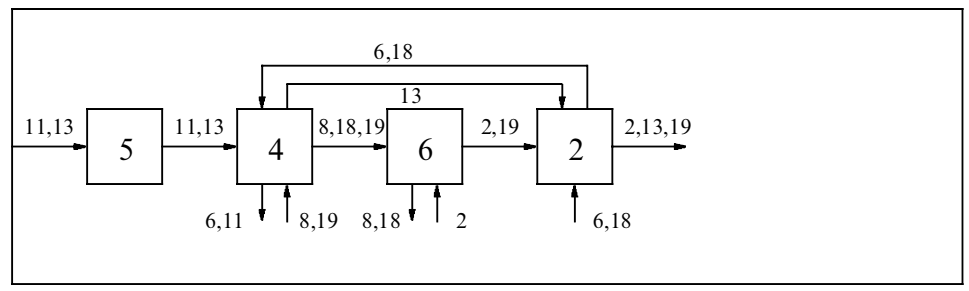

CELL 3

Figure 1. Material flow within cells.

nodes denote the machine types. As we can see from the figure, all the cells are independent at the end of the second stage.

Since there is an additional machine investment, allowing inter-cell movements may decrease the additional machine investment cost and simplify the material flow within the cell. The final solution obtained at the end of stage 2 is taken as an initial solution for the third stage, and we try to improve the objective value. The local search begins with finding alternatives. Parts, which are not making inter-cell moves and using the machines that should be bought, are candidates to make an inter-cell movement. Additional machine investment cost is made for machines 1,2 and 5 . Parts $1,2,3,4,5,6,7,11,12,13,14,15,16,17,18,19$ and 20 are using these machines and they can make an inter-cell movement. For example, part 13 is processed in cell 3 with its second route. If one of the machines in its routing is used only by part 13, then this machine can be removed from cell 3 and the operation on this machine can be performed in another cell having the required capacity. But there is no such machine in cell 3 . The same part can be assigned to another cell and/or can use another routing. Let's take the first routing of part 13, which is $\{2,6,4\}$, and look if it can be assigned to cell 2 . In cell 2 , machine 2 does not exist. Also, the capacity of machine 4 is not enough, hence two new machines are needed. $\left(N O P_{i}-\#\right.$ machines needed) $/ N O P_{i}=0.33$ is greater than $\theta=0.3$. Therefore, part 13 can be assigned to cell 2 and make an inter-cell movement to cell 1 for the operations on machines 2 and 4 , denoted as alternative $(13,1,2,1)$. All parts and cells are evaluated in the same way to find alternative solutions. $\Delta O b j$ for all alternatives, $(i, r r, j j, j j j)$, are calculated. The restricted candidate list is formed with $\max _{i, r, j, j, j j}\left\{\Delta O b j_{i, r r, j j, j j j}\right\}$ of -73.75 and Obj of $9314 . \mu$ is again taken as 0.1 . $(1,2,3,2),(2,1,2,1),(6,1,2,1),(13,2,1,2),(13,2,2,1)$, 
$(14,1,3,2),(15,1,1,3),(16,1,2,3)$ and $(16,1,3,2)$ form the set of alternatives in candidate list with $\triangle O b j$ values of $-982.5,-833.75,-125.25,-885,-885,-73.75$, $-287.5,-397.5$ and -397.5 , respectively. Since $\Delta O b j=-1902$ for alternative $(13,1,2,1)$, it is not included to the RCL. Suppose that $(14,1,3,2)$ is selected randomly, that means part 14 using route 1 is assigned to cell 3 and allowed to make an inter-cell movement to cell 2. All other part and route assignments remain the same. The new objective function value becomes $O b j=9314-73.75=9240.25$.

The local search continues until no alternative is left. The search is repeated for a fixed number of iterations to find different solutions to the problem. While performing all these steps, the solution giving the best objective value is kept as the incumbent solution. The final solution at the end of stage 3 is given in table 6 , where the additional MIC is 4502 and the new $O b j$ is 9316.5. Part 14 makes an inter-cell movement between cells 3 and 2, whereas part 15 between cells 1 and 3, and part 12 between cells 3 and 1 . The change in layouts and the material flow of parts in cells can be seen in figure 2. In this solution, the cells have an interaction with each other.

\begin{tabular}{lll}
\hline Cell & Layout & \multicolumn{1}{c}{ Parts $(i, r)$} \\
\hline 1 & 15623 & $(1,2)(3,1)(4,1)(10,1)(15,1)(16,1)(17,1)(20,1)$ \\
2 & 143 & $(5,1)(7,2)(9,1)$ \\
3 & 5462 & $(2,1)(6,1)(8,1)(11,2)(12,1)(13,2)(14,1)(18,2)(19,2)$ \\
\hline
\end{tabular}

Table 6. Solution obtained at the end of stage 3.

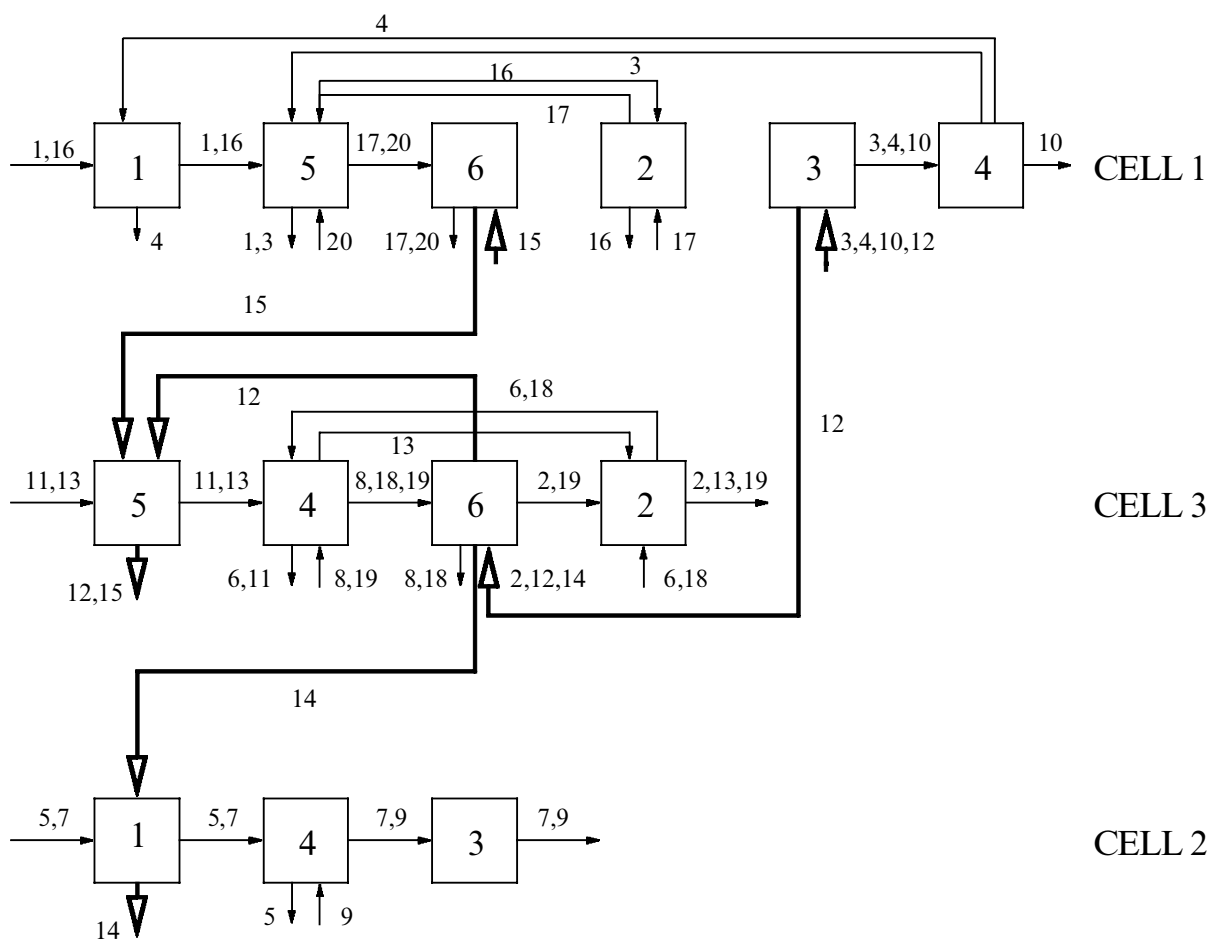

Figure 2. Material flow within and between cells at the end of stage 3 . 
The places of cells 2 and 3 are interchanged to decrease the material flow of parts 12 , 14 and 15 as described in step 3.7. Allowing inter-cell movements reduced the additional machine investment cost from 6074 to 4502 , hence the total profit is increased to 9316.5 .

\section{Experimental design}

We use the $\mathrm{C}$ language to code the proposed algorithm. The relaxed MIP subproblem is solved using CPLEX MIP solver. The code is compiled with Gnu C compiler and the problem is solved on a Sparc Station 10 under SunOS 5.4. There are five experimental factors that can affect the efficiency of the proposed algorithm, which are listed in table 7. Factor A, the number of parts and machine types, determines the size of the problem. Factor $\mathrm{B}$, the production cost, affects the route selection decisions. Factor $\mathrm{C}$ is the $h / C_{1}$ ratio, where $h_{i}$ is the intra-cell material handling cost per unit and $C_{i, 1}$ is the unit production cost of part $i$ per operation when it is produced with its first route such that $C_{i, 1}=\sum_{k \in M_{i, 1}} c_{i, 1, k} \cdot t_{i, 1, k} / N O P_{i}$. In order to evaluate the tradeoffs between the material handling costs and acquiring new machines, the variability of additional machine investment cost is used as another factor, Factor D. The fifth factor, Factor E, corresponds to the $H / h$ ratio, where $H_{i}$ is the inter-cell material handling cost per unit.

In this study, there are different ways of solving the proposed problem: acquiring additional machines or allowing intra-cell moves to retain cell independency, or using alternative routing, or allowing inter-cell moves, or any combination of these strategies. For a given set of cost parameters, tradeoffs can be made among these alternative strategies. Therefore, we choose the cost parameters as experimental factors at two different levels to see their influence on the computational results. Moreover, the same material handling equipment usually performs both the intracell and inter-cell moves for a certain part. We select these material handling cost terms in a comparable way $\left(H_{i}>h_{i}\right)$ so that we can evaluate different strategies in the proposed algorithm.

Since there are five factors and two levels, our experiment is a $2^{5}$ full-factorial design, which corresponds to thirty-two treatment combinations. The number of replications of each combination is taken as three producing 96 different randomly generated runs. Other variables in the system are treated as fixed parameters and summarized in table 8 , where $\mathrm{UN} \sim[a, b]$ represents a uniformly distributed random variable in interval $[a, b]$. The number of available machines are determined by considering the machines' capacities needed to produce all the parts with their first routings. It is known that cell formation problem is sensitive to the maximum number of cells. Therefore, we set $p$ to a reasonably high value so that it does not

\begin{tabular}{cccc}
\hline Factors & Definition & Level 1 & Level 2 \\
\hline A & $(\mathrm{n}, \mathrm{m})$ & $(20,6)$ & $(40,10)$ \\
$\mathrm{B}$ & Production cost & $\mathrm{UN} \sim[2.5,3.5]$ if $r=1$ & $\mathrm{UN} \sim[2.0,4.0]$ if $r=1$ \\
& $c_{\text {irk }}$ & $c_{i, 2,[k]}=1.2 \cdot c_{i, 1,[k]}$ if $r=2$ & $c_{i, 2,[k]}=1.2 \cdot c_{i, 1,[k]}$ if $r=2$ \\
$\mathrm{C}$ & $h / C_{1}$ ratio & 0.3 & 0.6 \\
$\mathrm{D}$ & Additional MIC & $\mathrm{UN} \sim[1400,1600]$ & $\mathrm{UN} \sim[400,2600]$ \\
$\mathrm{E}$ & $\mathrm{H} / \mathrm{h}$ ratio & 1.25 & 1.5 \\
\hline
\end{tabular}

Table 7. Experimental design factors. 


\begin{tabular}{ll}
\hline Parameters & Set of Values \\
\hline Number of operations, $N O P_{i}$ & $\mathrm{UN} \sim[2,3]$ \\
Number of routes, $R_{i}$ & $\mathrm{UN} \sim[1,2]$ \\
Processing time, $t_{i r k}$ & $\mathrm{UN} \sim[1,5]$ \\
Demand, $D_{i}$ & $\mathrm{UN} \sim[100,200]$ \\
Number of available machines, $M A_{k}$ & $\mathrm{UN} \sim\left[\rho_{k}, \rho_{k}+1\right]$ if $\rho_{k}=0,1$ \\
& $\mathrm{UN} \sim\left[\rho_{k}-1, \rho_{k}+1\right]$, otherwise \\
& where $\rho_{k}=\left\lceil\frac{\sum_{i} D_{i} t_{i 1 k}}{A_{k}}\right]$ \\
Cell size, $C S_{j}$ & 6 \\
Maximum number of cells, $p$ & $\left\lceil\frac{\sum_{k} M A_{k}}{6}\right\rceil+3$ \\
Available machine capacity, $A_{k}$ & $2000 \mathrm{~min} / \mathrm{week}$ \\
Minimum utilization level, $\alpha_{k j}$ & $0.1 * A_{k}$ \\
Selling price, $S P_{i}$ & $1.30 \cdot \sum_{k \in M R_{i 1}} t_{i 1 k} \cdot c_{i 1 k}$ \\
Raw material cost, $R M_{i}$ & $0.05 \cdot \sum_{k \in M R_{i 1}} t_{i 1 k} \cdot c_{i 1 k}$ \\
Minimum profit level, $L P_{j}$ & $0.25 \cdot D_{i}\left(S P_{i}-R M_{i}-\sum_{k \in M R_{i 1}} c_{i 1 k} \cdot t_{i 1 k}\right) / p$ \\
\hline
\end{tabular}

Table 8. Fixed parameters.

\begin{tabular}{|c|c|c|c|c|c|c|c|c|}
\hline \multirow{2}{*}{$\frac{(\mathrm{BCD})}{(000)}$} & \multirow{2}{*}{$\frac{\text { Run }}{01}$} & \multicolumn{2}{|c|}{ R SP Objective } & \multicolumn{2}{|c|}{ Final Solution } & \multicolumn{2}{|c|}{ UB* or OPT } & \multirow{2}{*}{$\frac{\% \text { GAP }}{0.00 \%}$} \\
\hline & & 26182 & $(4.5)$ & 24677 & (1872.6) & 24677 & $(5639.2)$ & \\
\hline & 02 & 14593 & $(53.6)$ & 13975 & (194.7) & 13975 & (314.9) & $0.00 \%$ \\
\hline & 03 & 16093 & (903.8) & 15821 & (276.6) & $15900 *$ & $(4264.9)$ & $0.50 \% *$ \\
\hline \multirow{3}{*}{$(001)$} & 04 & 26182 & (5.7) & 24677 & $(2362.3)$ & 24677 & (6195.1) & $0.00 \%$ \\
\hline & 05 & 15189 & $(25.8)$ & 13744 & $(162.9)$ & $14947^{*}$ & $(145.1)$ & $8.05 \% *$ \\
\hline & 06 & 16069 & (825.7) & 15797 & (391.5) & $15876^{*}$ & $(3907.3)$ & $0.50 \% *$ \\
\hline \multirow[t]{3}{*}{$(010)$} & 07 & 23588 & (130.6) & 21109 & (724.8) & 21154 & (15 367.7) & $0.21 \%$ \\
\hline & 08 & 10392 & (165.5) & 9314 & (111.8) & $10143^{*}$ & (951.9) & $8.17 \% *$ \\
\hline & 09 & 12810 & $(2400.8)$ & 11175 & $(86.5)$ & $12175^{*}$ & $(16805.9)$ & $8.21 \% *$ \\
\hline \multirow[t]{3}{*}{$(011)$} & 10 & 23588 & $(95.2)$ & 21156 & (987.5) & 21467 & $(3590.0)$ & $1.45 \%$ \\
\hline & 11 & 10571 & (125.5) & 9716 & (145.1) & $10571 *$ & $(308.8)$ & $8.09 \% *$ \\
\hline & 12 & 12786 & $(2980.1)$ & 11151 & $(79.9)$ & $12402 *$ & (11 463.8) & $10.09 \% *$ \\
\hline \multirow[t]{3}{*}{$(100)$} & 13 & 26332 & $(4.1)$ & 24700 & $(1970.4)$ & 24798 & $(5574.2)$ & $0.40 \%$ \\
\hline & 14 & 13648 & $(75.3)$ & 13083 & $(97.5)$ & 13083 & $(425.6)$ & $0.00 \%$ \\
\hline & 15 & 15962 & (494.4) & 15672 & $(262.3)$ & $15774^{*}$ & $(4029.9)$ & $0.65 \%$ * \\
\hline \multirow[t]{3}{*}{ (101) } & 16 & 26332 & $(3.8)$ & 24700 & $(2344.2)$ & $24798 *$ & $(7224.8)$ & $0.40 \%$ * \\
\hline & 17 & 14255 & $(10.5)$ & 12700 & (161.9) & $14047^{*}$ & $(103.3)$ & $9.59 \% *$ \\
\hline & 18 & 15938 & $(502.9)$ & 15648 & $(1033.7)$ & $15750 *$ & $(4320.8)$ & $0.65 \%$ * \\
\hline \multirow[t]{3}{*}{$(110)$} & 19 & 23672 & (223.3) & 21074 & $(737.0)$ & $21549 *$ & $(10785.3)$ & $2.20 \%$ * \\
\hline & 20 & 9476 & (259.7) & 8484 & (106.5) & $9203 *$ & $(1122.0)$ & $7.81 \%$ * \\
\hline & 21 & 12490 & $(5172.2)$ & 12490 & $(17.5)$ & 12490 & $(6700.0)$ & $0.00 \%$ \\
\hline \multirow[t]{3}{*}{ (111) } & 22 & 23672 & $(124.2)$ & 21337 & $(1022.2)$ & 21637 & $(6393.4)$ & $1.39 \%$ \\
\hline & 23 & 9561 & $(105.5)$ & 8835 & $(116.8)$ & $9561 *$ & (302.4) & $7.59 \% *$ \\
\hline & 24 & 12916 & $(2122.6)$ & 12336 & (5.1) & 12466 & $(7972.9)$ & $1.04 \%$ \\
\hline
\end{tabular}

\footnotetext{
* Deviation from the upper bound.
}

Table 9. 20 parts and 6 machine types. 
become a tight constraint. Furthermore, the machine types for the routings of parts are selected with certain probabilities. The parameters of the algorithm, $\theta, \mu$, maximum iteration number and maximum step size are selected as 0.3, 0.1, 100 and 100, respectively, after a number of trial runs.

The relative difference between the objective function value obtained by the algorithm and an upper bound or optimal solution to the originally proposed MIP model, denoted as percentage gap, and the computation time are used as performance measures in this experimental design. These performance measures will be analysed separately for two main parts of the algorithm. In the first part of the algorithm, inter-cell movements are not allowed, hence the first four factors are used. For this part, the results of all runs for 20 part types and 6 machine types can be seen in table 9. 'R SP objective' is the objective function value obtained by solving the relaxed subproblem, which provides an upper bound to the main problem. 'Final solution' is the objective function value obtained at the end of the second stage. The values in the parentheses are the corresponding computation times in seconds. The values in the next column of the same table, 'UB* or OPT', are the new upper bounds or the optimal solutions for the MP. If we cannot find an optimum solution in a reasonable computation time then we add the following constraints $\beta_{k l j}+\beta_{l k j} \leq 1 \forall k, l, j$ to the subproblem to obtain a tighter upper bound. The last column in the table is the percentage gap.

When we analyse all the results, we know the optimum solution in 10 out of 24 runs. In 5 of them, the proposed algorithm also finds the optimum value. In the other five runs, the average gap is $0.332 \%$ with a minimum of $0.21 \%$ and a maximum of $1.45 \%$. The average gap from the optimum value for these 10 runs is $0.116 \%$. This means that the solution found by the algorithm is very close to the optimum value. In the other 14 runs, the optimum solution could not be found, but a new upper bound is obtained for MP. The average gap from the upper bound for these 14 runs is $5.18 \%$. The gaps are small when we consider the difficulty of solving the MP in a reasonable computation time. The average computation time is 700 seconds for the first stage, 636 seconds for the second stage with a total of 1336 seconds. The constraints added to the RSP increase the computation time to an average of 5177 seconds, which is four times larger than the time needed by the proposed algorithm. Also, solving the RSP with additional constraints does not guarantee a feasible solution to the MP. But, the algorithm guarantees a feasible solution which is very close to the optimum value in all cases.

The results of the runs for 40 parts and 10 machine types can be seen in table 10 . As the size of the problem increases, solving the RSP optimally in a reasonable computation time becomes more difficult. So, a predetermined time limit of 10800 seconds is used for the first stage of the algorithm to find a feasible integer solution for the RSP. Since this solution may not be optimal, we cannot say whether it is an upper bound for the MP or not. Also, this solution is infeasible for the MP. The objective function values of the solutions found at the end of 10800 seconds for the first stage can be seen in the third column, 'RSP objective'. When the time limit is reached, the best LP relaxation value found up to that time is shown in the next column. This can be thought as an upper bound, but a loose one. The objective function values found by the R SP are smaller than the values found by the proposed algorithm, because the optimal solutions cannot be found by the RSP within the predetermined time limit. In the last column, the percentage difference between the objective function values of the relaxed subproblem and the algorithm can be found. 


\begin{tabular}{|c|c|c|c|c|c|c|c|c|}
\hline \multirow{2}{*}{$\frac{(\mathrm{BCD})}{(000)}$} & \multirow{2}{*}{$\frac{\text { Run }}{01}$} & \multicolumn{2}{|c|}{ RSP Objective } & \multicolumn{2}{|c|}{ Best LP Relaxation } & \multicolumn{2}{|c|}{ Final Solution } & \multirow{2}{*}{$\frac{\text { Improvement }}{75.0 \%}$} \\
\hline & & 9866 & $(10800)$ & 30050 & $(10800)$ & 17260 & (1358) & \\
\hline & 02 & 15183 & $(10800)$ & 30433 & $(10800)$ & 23464 & $(3967)$ & $54.5 \%$ \\
\hline & 03 & 23424 & $(10800)$ & 30927 & $(10800)$ & 23781 & $(1016)$ & $1.5 \%$ \\
\hline \multirow[t]{3}{*}{$(001)$} & 04 & 12771 & $(10800)$ & 28661 & $(10800)$ & 19153 & $(2125)$ & $50.0 \%$ \\
\hline & 05 & 8893 & $(10800)$ & 29876 & $(10800)$ & 22500 & $(5940)$ & $153.0 \%$ \\
\hline & 06 & 20355 & $(10800)$ & 31448 & $(10800)$ & 22172 & $(5466)$ & $8.9 \%$ \\
\hline \multirow[t]{3}{*}{$(010)$} & 07 & 2553 & $(10800)$ & 23632 & $(10800)$ & 7262 & (2039) & $184.4 \%$ \\
\hline & 08 & 10784 & $(10800)$ & 24862 & $(10800)$ & 17439 & (3448) & $61.7 \%$ \\
\hline & 09 & 3958 & $(10800)$ & 24829 & $(10800)$ & 12248 & $(16063)$ & $209.4 \%$ \\
\hline \multirow[t]{3}{*}{$(011)$} & 10 & 2217 & $(10800)$ & 22997 & $(10800)$ & 10672 & $(14823)$ & $381.4 \%$ \\
\hline & 11 & -2477 & $(10800)$ & 24678 & $(10800)$ & 12203 & $(3863)$ & $592.6 \%$ \\
\hline & 12 & 2202 & $(10800)$ & 25595 & $(10800)$ & 18127 & (3019) & $732.2 \%$ \\
\hline \multirow[t]{3}{*}{$(100)$} & 13 & 8015 & $(10800)$ & 30558 & $(10800)$ & 16233 & $(603)$ & $102.5 \%$ \\
\hline & 14 & 15422 & $(10800)$ & 29727 & $(10800)$ & 23538 & $(13898)$ & $46.2 \%$ \\
\hline & 15 & 26052 & $(10800)$ & 30970 & $(10800)$ & 25019 & (2394) & $-4.0 \%$ \\
\hline \multirow[t]{3}{*}{ (101) } & 16 & 17587 & $(10800)$ & 29058 & $(10800)$ & 18362 & (4066) & $4.4 \%$ \\
\hline & 17 & 14594 & $(10800)$ & 29048 & $(10800)$ & 22547 & (4872) & $54.5 \%$ \\
\hline & 18 & 28582 & $(10800)$ & 31455 & $(10800)$ & 26752 & $(20674)$ & $-6.4 \%$ \\
\hline \multirow[t]{3}{*}{ (110) } & 19 & 1656 & $(10800)$ & 24635 & $(10800)$ & 7564 & (2149) & $356.8 \%$ \\
\hline & 20 & 3778 & $(10800)$ & 24205 & $(10800)$ & 17210 & $(15018)$ & $355.5 \%$ \\
\hline & 21 & 6073 & $(10800)$ & 25077 & $(10800)$ & 13637 & (8939) & $124.6 \%$ \\
\hline \multirow[t]{3}{*}{$(111)$} & 22 & 8159 & $(10800)$ & 23979 & $(10800)$ & 13162 & $(14325)$ & $61.3 \%$ \\
\hline & 23 & 10431 & $(10800)$ & 24160 & $(10800)$ & 14850 & $(708)$ & $42.4 \%$ \\
\hline & 24 & 4282 & $(10800)$ & 25940 & $(10800)$ & 16141 & $(34806)$ & $276.9 \%$ \\
\hline
\end{tabular}

Table 10. 40 parts and 10 machine types.

This percentage shows the improvement in the objective function value. When all the results are analysed, it can be seen that a feasible solution cannot be found to any RSP in the first stage. The solution found at the end of first stage is improved in almost all cases by the algorithm, and the average improvement is $163 \%$. The average computation time for the second stage is 7732 seconds. Although the computation times are relatively large, they are much less than the planning horizon for such a strategic level long-term planning decision.

In the second part of the algorithm, the inter-cell movements are introduced to the problem. The fifth factor, $H / h$ ratio, is included to the experimental design. The inter-cell movements directly increase the measurable material handling cost between cells. We know that the inter-cell moves complicate the controlling, planning and scheduling activities in a cell, but it is very difficult to estimate their intangible impact on the production cost besides the direct material handling cost. Although we only consider the measurable handling costs in our experimental design, the intangible costs can be included by relatively increasing the $H / h$ ratio. But when this ratio is large, inter-cell moves will be undesirable compared to other alternative strategies, consequently inter-cell movement may not be allowed in order not to violate the low profit level constraint. The intangible costs can also be included while determining the layout of the factory by using the CRAFT algorithm by specifying different cost parameters for the inter-cell movements in the from-to-chart as discussed in Step 3.7. In the second part of the algorithm, we take the final solution found in the first part as an initial solution and try to improve it for a number of iterations. The average computation time is 362 seconds for the second part. 


\begin{tabular}{llrrrrrr}
\hline Factor & Level & TMHC & \multicolumn{1}{c}{ TVPC } & TSPR M & TPR & MIC & Objective \\
\hline A & Low & 4657.1 & 62513.3 & 85142.9 & 17976.2 & 1990.8 & 15981.9 \\
& High & 11762.1 & 132784.1 & 171400.7 & 26915.1 & 8213.7 & 18698.3 \\
B & Low & 8067.8 & 98074.9 & 128767.7 & 22578.0 & 5294.8 & 17283.8 \\
& High & 8351.4 & 97222.5 & 127775.9 & 22313.3 & 4909.7 & 17396.4 \\
C & Low & 6332.5 & 97443.2 & 128273.7 & 24451.1 & 4098.2 & 20353.5 \\
& High & 10086.8 & 97854.2 & 128269.9 & 20440.3 & 6106.4 & 14326.7 \\
D & Low & 8184.5 & 97720.9 & 128269.9 & 22353.3 & 5009.9 & 17338.3 \\
& High & 8234.7 & 97576.5 & 128273.7 & 22538.1 & 5194.6 & 17341.9 \\
E & Low & 8318.0 & 97645.9 & 128273.7 & 22363.5 & 4944.9 & 17419.2 \\
& High & 8101.3 & 97651.5 & 128269.9 & 22527.8 & 5259.7 & 17261.0 \\
\hline
\end{tabular}

Table 11. Average cost values for low and high level of each factor.

We now analyse the cost terms in the objective function and the objective function value to see the individual effect of the experimental factors on these terms. The cost terms in the objective function are total material handling cost (TMHC), total variable production cost (TVPC), difference between total revenue and raw material cost (TSPR M), the sum of individual profits of cells without considering additional machine investment cost (TPR), and the additional machine investment cost (MIC). The cost terms for each level of each factor can be seen in table 11 . The values in each row are the average values of 48 runs out of 96 runs for the low and high levels of experimental design factors. The number of parts and machine types have an high effect on all the cost terms. As the size of the system increases, total material handling, production and raw material costs and revenue increases due to the high production volume. Total profit of cells also increases. Total additional machine investment cost increases due to either insufficient machine capacity or high material flow in cells. The increase in production cost variability does not affect any of the performance measures significantly. This may be due to the high variability in processing times of parts on machines. There is a direct relationship between material handling cost and total additional machine investment cost. When the material handling costs increase, buying new machines becomes more profitable. Intercell material handling cost has a significant impact on total material handling and additional machine investment costs. When inter-cell material handling cost is low, more parts can make an inter-cell movement, which decreases the additional machine investment cost and increases the total profit as expected.

\section{Conclusion}

In this study, the part-family and machine-cell formation problems are solved with the within-cell layout problem simultaneously. The proposed approach has an advantage of allowing more accurate portrayal of the operation of CM systems by using production volumes, processing times, operation sequences and alternative routes to assess the impact of capacity constraints. To the best of our knowledge, this is the first study that considers the efficiency of both individual cells and the overall system in monetary terms. Each cell should make at least a certain amount of profit to attain self-sufficiency, while we maximize the total profit of the system using a holonistic approach.

An MIP model, under routing, layout, cell size, low utilization and low profit level constraints, is formulated to solve the PFMCF and within cell layout problems 
simultaneously to maximize the total profit. We also propose a local search algorithm, which provides two alternative solutions; one with independent cells and the other one with inter-cell movement. The results of the experimental design are very encouraging. The proposed algorithm always finds a feasible solution to the problem in a reasonable computation time. When the size of the problem is small, the average gap is only $0.116 \%$ for the 20 part and 6 machine type problems for which we know the optimum values. The computation times of the algorithm are significantly less than the computation times needed to find tight upper bounds to the main problem. When the problem size increases, a tight upper bound cannot be found for comparison purposes. But, the objective function value obtained at the end of first stage is improved significantly with an average of $163 \%$. Since this problem is a strategic level long-term design problem, the run times are much less than the planning horizon.

\section{References}

Adil, G. K., Rajamani, D. and Strong, D., 1996, Cell formation considering alternate routings. International Journal of Production Research, 34, 1361-1380.

Akturk, M. S. and BAlKose, H. O., 1996, Part machine grouping using a multi-objective cluster analysis. International Journal of Production Research, 34, 2299-2315.

ASKIN, R. and ZHOU, M., 1998, Formation of independent flow-line cells based on operation requirements and machine capabilities. IIE Transactions, 30, 319-329.

BAZARGAN-LARI, M. and KAEBERNICK, H., 1996, Intracell and intercell layout designs for cellular manufacturing. International Journal of Industrial Engineering, 3, 139-150.

BeAulieu, A., GHARBI, A. and AIT-KADI, D., 1997, An algorithm for cell formation and machine selection problems in the design of a cellular manufacturing system. International Journal of Production Research, 35, 1857-1874.

ChoobineH, F., 1988, A framework for the design of cellular manufacturing systems. International Journal of Production Research, 26, 1161-1172.

DAHEL, N. E., 1995, Design of cellular manufacturing system in tandem configuration. International Journal of Production Research, 33, 2079-2095.

Heragu, S. S. and Gupta, Y. P., 1984, A heuristic for designing cellular manufacturing facilities. International Journal of Production Research, 32, 125-140.

Ho, Y. C. and MoodiE, C. L., 1996, Solving cell formation problems in a manufacturing environment with flexible processing and routing capabilities. International Journal of Production Research, 34, 2901-2923.

HöPf, M., 1994, Holonic manufacturing systems. CIM-Europe Annual Conference, Copenhagen, pp. 84-93.

Joines, J. A., CUlbreth, C. T. and King, R. E., 1996, Manufacturing cell design: an integer programming model employing genetic algorithms. IIE Transactions, 28, 69-85.

Offodile, O. F., MehreZ, A. and GrZnAR, J., 1994, Cellular manufacturing: A taxonomic review framework. Journal of Manufacturing Systems, 13, 196-219.

VAKHARIA, A. J. and CHANG, Y.-L., 1997, Cell formation in group technology: A combinatorial search approach. International Journal of Production Research, 35, 2025-2043.

VAKHARIA, A. J. and WEMMERLÖV, U ., 1990, Designing a cellular manufacturing system: A materials flow approach based on operation sequences. IIE Transactions, 22, 84-97.

Verma, P. and Ding, F.-Y., 1995, A Sequence-based materials flow procedure for designing manufacturing cells. International Journal of Production Research, 33, 3267-3281. 